\title{
Influence of the cosmological constant on gravitational lensing in small systems
}

Sereno, M

\begin{abstract}
The cosmological constant Lambda affects gravitational lensing phenomena. The contribution of Lambda to the observable angular positions of multiple images and to their amplification and time delay is here computed through a study of the weak deflection limit of the equations of motion in the Schwarzschild-de Sitter metric. Because of Lambda the unresolved images are slightly demagnified, the radius of the Einstein ring decreases, and the time delay increases. The effect is however negligible for near lenses. In the case of a null cosmological constant, we provide some updated results on lensing by a Schwarzschild black hole
\end{abstract}

DOI: https://doi.org/10.1103/PhysRevD.77.043004

Posted at the Zurich Open Repository and Archive, University of Zurich ZORA URL: https://doi.org/10.5167/uzh-16762

Journal Article

Accepted Version

Originally published at:

Sereno, M (2008). Influence of the cosmological constant on gravitational lensing in small systems. Physical Review D, 77(4):043004.

DOI: https://doi.org/10.1103/PhysRevD.77.043004 


\title{
On the influence of the cosmological constant on gravitational lensing in small systems
}

\author{
Mauro Sereno* \\ Institut für Theoretische Physik, Universität Zürich, Winterthurerstrasse 190, CH-8057 Zürich, Switzerland.
}

(Dated: October 31, 2007)

\begin{abstract}
The cosmological constant $\Lambda$ affects gravitational lensing phenomena. Following a study of the equations of motion of the Schwarzschild-de Sitter metric in the weak deflection limit, the contribution of $\Lambda$ to the observable angular positions of multiple images and to their amplification and time delay is computed. Due to $\Lambda$ the unresolved images are slightly demagnified, the radius of the Einstein ring decreases and the time delay increases. The effect is however negligible for near lenses.
\end{abstract}

PACS numbers: 95.30.Sf, 04.70.Bw, 98.62.Sb

Keywords: Classical black holes; Gravitational Lensing

\section{INTRODUCTION}

The interpretation of the cosmological constant $\Lambda$ is a very fascinating and traditional topic in theoretical physics. On the observational side, large scale structure observations have made a strong case for $\Lambda$ as a possible choice for dark energy. In fact, a very small value of $\Lambda \sim 10^{-52} \mathrm{~m}^{-2}$, together with dark matter, can provide a suitable framework for observational cosmology [1].

The consideration that the cosmological constant should take part in phenomena on every physical scale has stimulated many investigations on very different scale-lengths. Despite no convincing method for constraining $\Lambda$ in an Earth's laboratory has been proposed [2], local astronomical phenomena seem to be more promising. The cosmological constant can influence the motion of massive bodies [3-5] and the effect on the perihelion precession of solar system planets together with other solar and stellar tests has been considered to put an upper bound of $\Lambda \lesssim 10^{-42} \mathrm{~m}^{-2}$ [4, 6-8, and references therein]. The cosmological constant also affects the gravitational equilibrium of large astrophysical structures $[9,10]$ and could have observable consequences by producing lower velocity dispersion around the Hubble flow on the scale of the Local Volume [11].

Recently, Rindler and Ishak [12] discussed how the cosmological constant takes part in gravitational lensing. Taking into account $\Lambda$ through the Schwarzschild-de Sitter (SdS) metric, they showed that even if the exact differential equation for a light path in the coordinate space can be written in a form that does not involve $\Lambda$ [3], the cosmological constant contributes to the bending of light through the metric itself, which determines the actual observations that can be made on the orbit equation. In fact, one must consider not only the null geodesic equation but also the process of measurement [13, 14].

Following this correction of the long-standing misconception that $\Lambda$ does not affect the observed deflection angle, in this paper I further investigate the effect of the cosmological constant in gravitational lensing observations in near systems in the approximation of the weak deflection limit. The lens equation is derived from the lightlike null geodesics of the

\footnotetext{
*Electronic address: sereno@physik.unizh.ch
}

SdS metric. Results are expressed in terms of the invariants of the light ray, avoiding ambiguities connected to coordinatedependent quantities [15, 16], and of observable quantities. The weak deflection limit allows a clear insight on the effect of $\Lambda$ but it is to be remarked that a gravitational lens equation without approximations can be written in generic spherically symmetric and static spacetimes [17].

The paper is organized as follows. In Sec. II, the null orbits are solved in the weak deflection limit. In Sec. III the lens equation is first written in terms of the observed angle and then solved with a perturbation method. The image amplification and time delay are discussed in Sec. IV and V, respectively. Some quantitative estimates on the effect of $\Lambda$ are illustrated in Sec. VI and finally Section VII is devoted to some considerations.

\section{GEODESIC EQUATION}

The effect of $\Lambda$ on gravitational lensing can be considered in the framework of the spherically symmetric Schwarzschild vacuum solution with a cosmological constant, also known as Schwarzschild-de Sitter (SdS) or Kottler space-time [18],

$$
d s^{2}=f_{\Lambda}(r) d t^{2}-\frac{d r^{2}}{f_{\Lambda}(r)}-r^{2}\left(d \theta^{2}-\sin ^{2} \theta d \phi^{2}\right),
$$

where

$$
f_{\Lambda}(r) \equiv\left(1-\frac{2 m}{r}-\frac{\Lambda r^{2}}{3}\right),
$$

and $m$ is the black hole mass. We are using units $G=c=1$. For radii approaching $r_{\Lambda} \equiv \sqrt{3 / \Lambda}$, a coordinate singularity, i.e. the de Sitter horizon, occurs. Due to spherical symmetry, photon trajectories can be conveniently restricted to the central $\theta=\pi / 2$ plane. We consider the standard framework of gravitational lensing in the weak deflection limit, where the source of radiation and the observer are remote from the lens. Lensing in a static, spherically symmetric metric is usually investigated considering the case of a asymptotically flat spacetime under the assumption of both observer and source in this regime [15]. Here, we have to consider a region of spacetime which is well inside the outer horizon and where the intrinsic 
geometry of the 2-metric of the equatorial plane $\theta=\pi / 2$ undergoes a transition from a nearly Flamm paraboloid of revolution in the inner region, as typical of the Schwarzschild metric, to a spherical geometry of radius $r_{\Lambda}$ in the very outer nearly de Sitter spacetime $[12,19]$. Since the observer lies in this curved region of spacetime, even if the null geodesics are formally indistinguishable from the $\Lambda=0$ case in the coordinate space, the observable quantities will be affected by the cosmological constant [12].

In the following analysis the observer and the emitter are taken to be static. The observer coordinates are denoted $\left\{r_{\mathrm{o}}, \phi_{\mathrm{o}}=0\right\}$, where $\phi_{\mathrm{o}}$ has been fixed without loss of generality. The source coordinates are denoted as $\left\{r_{\mathrm{s}}, \phi_{\mathrm{s}}\right\}$. The orbital equation for a light ray from the source to the observer can then be written in terms of the first integral of motion $b\left(\equiv \dot{\phi} r^{2}\right)$ as

$$
\phi_{\mathrm{s}}= \pm \int \frac{d r}{r^{2}}\left(\frac{1}{b^{2}}+\frac{1}{r_{\Lambda}^{2}}-\frac{1}{r^{2}}+\frac{2 m}{r^{3}}\right)^{-1 / 2},
$$

where the sign of the integral is adhered to the sign of $d r$ and changes at the inversion points in the $r$-motion. Along its path from the source to the observer, the photon passes by the black hole at a minimum distance $r_{\min }$ which is much larger than the gravitational radius. In the weak deflection limit, this closest approach is the only turning point in the $r$-motion. Defining a new constant $b_{\Lambda}$ such that $1 / b_{\Lambda}^{2}=\left(1 / b^{2}+1 / r_{\Lambda}^{2}\right)$, we can see as the geodesics are formally identical to those in a Schwarzschild spacetime without cosmological constant. This can be seen even more clearly taking the second derivative $d^{2} r / d \phi^{2}$, which eliminates $\Lambda$ from the equation. Equation (3) can be solved in terms of elliptical functions [13] and exact analytical results can be obtained even considering a spinning black hole [20]. For an asymptotically flat spacetime, $b$ can be viewed as the impact parameter.

Even if the equations of motion for either a massive test particle or a photon can be solved exactly [13, 20], expression are quite involved, so that to have an insight on the lensing observables it can be useful to treat the geodesic motion using a perturbation approach. A fundamental assumption in the weak deflection limit is that the point of closest approach lies well outside the gravitational radius, i.e. $m / b \equiv \epsilon_{m} \ll 1$. The observer and the source lie very far from the lens. It can be shown that $b / r_{\mathrm{O}} \sim b / r_{\mathrm{s}} \sim \epsilon_{m}$ [15]. Furthermore, we assume that the system is embedded in a region well inside the outer horizon, $r_{\mathrm{o}}, r_{\mathrm{S}} \ll r_{\Lambda}$. In what follows, we will expand quantities of interest according to the expansion parameters $\epsilon_{m}$ and $\epsilon_{\Lambda} \equiv r_{\mathrm{o}} / r_{\Lambda}$ but, for the sake of brevity, we will produce our results up to a given formal order in $\epsilon$, collecting terms coming from any combination of the two expansion parameters.

The light ray minimum radial distance $r_{\min }$ to the lens is determined by $r^{2}=b^{2} f_{\Lambda}(r)$, whose exact solution is known analytically [12]. Expanding the solution in the weak deflection limit as a power series in $\epsilon$ we find

$$
r_{\min } \simeq b\left\{1-\frac{m}{b}-\frac{3 m^{2}}{2 b^{2}}-\frac{4 m^{3}}{b^{3}}-\frac{105 m^{4}}{8 b^{4}}-\frac{b^{2}}{2 r_{\Lambda}^{2}}\right\} .
$$

An expression for the minimum approach including terms $\mathcal{O}\left(\epsilon^{4}\right)$ for the Kerr metric can be found in [21]. Equation (4) for the case of null cosmological constant agrees with the result in [15].

The integral in Eq. (3) can be solved approximately under the assumptions discussed above and following standard methods and procedures $[15,21]$. We get

$$
\begin{aligned}
\phi_{\mathrm{s}} & =-\pi-\frac{4 m}{b}+b\left(\frac{1}{r_{\mathrm{s}}}+\frac{1}{r_{\mathrm{o}}}\right)-\frac{15 m^{2} \pi}{4 b^{2}}-\frac{128 m^{3}}{3 b^{3}} \\
& +\frac{b^{3}}{6}\left(\frac{1}{r_{\mathrm{s}}^{3}}+\frac{1}{r_{\mathrm{o}}^{3}}\right)-\frac{3465 m^{4} \pi}{64 b^{4}}-\frac{3584 m^{5}}{5 b^{5}}-\frac{2 m b}{r_{\Lambda}^{2}} \\
& -\frac{m b^{3}}{4}\left(\frac{1}{r_{\mathrm{s}}^{4}}+\frac{1}{r_{\mathrm{o}}^{4}}\right)+\frac{3 b^{5}}{40}\left(\frac{1}{r_{\mathrm{s}}^{5}}+\frac{1}{r_{\mathrm{o}}^{5}}\right) \\
& -\frac{b^{3}}{2 r_{\Lambda}^{2}}\left(\frac{1}{r_{\mathrm{s}}}+\frac{1}{r_{\mathrm{o}}}\right)+\mathcal{O}\left(\epsilon^{6}\right) .
\end{aligned}
$$

The cosmological constant contributes to the geodesic equation through terms of order of $\mathcal{O}\left(\epsilon^{5}\right)$. The term $2 \mathrm{bm} / \mathrm{r}_{\Lambda}^{2}$, where neither the source or the observer radial position enters, can be considered as local. We are assuming the impact parameter $b$ to be positive.

\section{LENS EQUATION}

The lens equation is a mapping relating the angular position of the source and observed position of its images. It is usually given in terms of the apparent angular position of the image in the sky, i.e. the angle $\vartheta$ between the tangent to the photon trajectory at the observer and the radial direction to the black hole. The angle $\vartheta$ is strictly linked to the constant of motion through the relation

$$
\sin \vartheta=\sqrt{f_{\Lambda}\left(r_{\mathrm{o}}\right)} \frac{b}{r_{\mathrm{o}}}
$$

For small angles,

$$
\vartheta \simeq \frac{b}{r_{\mathrm{o}}}+\frac{b^{3}}{6 r_{\mathrm{o}}^{3}}\left[1-\frac{6 m r_{\mathrm{o}}}{b^{2}}-\frac{3 r_{\mathrm{o}}^{4}}{b^{2} r_{\Lambda}^{2}}\right] .
$$

Due to the presence of $\Lambda$, the relation between $b$ and the observed angle changes by a term of order $\mathcal{O}\left(\epsilon^{3}\right)$, two orders of magnitude higher than the contribution of $\Lambda$ to the variation of the coordinate azimuthal angle, see Eq. (5). This relation between the observed angle and the constant of motion determines the extent to which $\Lambda$ affects the lensing observables. In the following resolution of the lens equation, calculations will be then performed up to order $\mathcal{O}\left(\epsilon^{3}\right)$.

Once we use angular coordinates for the image positions instead on the invariants of motion, it can be appropriate to introduce a series expansion parameter in the weak deflection limit based on the angular Einstein ring defined through radial distances [21],

$$
\vartheta_{\mathrm{E}} \equiv \sqrt{4 m \frac{r_{\mathrm{s}}}{r_{\mathrm{o}}\left(r_{\mathrm{o}}+r_{\mathrm{s}}\right)}}
$$

the expansion parameter $\varepsilon_{\mathrm{E}}$ is then defined as $\varepsilon_{\mathrm{E}} \equiv \theta_{\mathrm{E}} / 4 D$ $[15,21]$ where $D \equiv r_{\mathrm{s}} /\left(r_{\mathrm{o}}+r_{\mathrm{s}}\right)$. As for the case of the 
geodesic equation, expansions are actually made in terms of two parameters, $\varepsilon_{\mathrm{E}}$ and $\varepsilon_{\Lambda} \equiv \epsilon_{\Lambda}$. Mixed terms are collected through a given formal order in the parameter $\varepsilon$.

It is customary in lensing studies to write the source position in terms of the angle $B$ at which the source would be seen in absence of the lens, i.e for $m=0$. In analogy with Eq. (6), $B$ is then given by $\sin B=\sqrt{1-\left(r_{\mathrm{o}} / r_{\Lambda}\right)^{2}} b_{\mathrm{s}} / r_{0}$ with $b_{\mathrm{s}}$ being a fictitious constant of motion which solves the geodesic equation Eq. (3) for the actual source and observer coordinates but for $m=0$. The azimuthal source coordinate, $\phi_{\mathrm{s}}$, can then be expressed in terms of $B$ plugging the unlensed constant $b_{\mathrm{s}}$ in Eq. (5). The lens equation in the form

$$
B=B(\vartheta ; m, \Lambda)
$$

is finally obtained by first writing $\phi_{\mathrm{s}}$ as a function of either $\vartheta$ and $B$ and then equating the two expressions. We will consider source positions $B \geq 0$. At the lowest order, $B \simeq D\left(\phi_{\mathrm{s}}+\pi\right)$.

The lens equation can be solved term by term. We assume that the solution can be written as a series in $\varepsilon$,

$$
\theta=\vartheta_{\mathrm{E}}\left\{\theta_{(0)}+\theta_{(1)} \varepsilon+\theta_{(2)} \varepsilon^{2}+\mathcal{O}\left(\varepsilon^{3}\right)\right\}
$$

The source position $B$ can be rescaled as $\beta=B / \vartheta_{\mathrm{E}}$. At first order, the lens equations take the standard form

$$
\beta=\theta_{(0)}-\frac{1}{\theta_{(0)}},
$$

with the usual pair of solutions

$$
\theta_{(0)}^{ \pm}=\frac{1}{2}\left(1 \pm \sqrt{1+\frac{4}{\beta^{2}}}\right) \beta
$$

The next order correction is

$$
\theta_{(1)}=\frac{15 \pi}{16\left(1+\theta_{(0)}^{2}\right)}
$$

Up to and including the second order corrections, the cosmological constant is ineffective and lensing is pure Schwarzschild. The cosmological constant shows up at the next order,

$$
\theta_{(2)}=\frac{8}{\theta_{0}\left(\theta_{0}^{2}+1\right)}\left[1+\theta_{0}^{2}-\theta_{0}^{4}+D\left(1-\frac{7 \theta_{0}^{2}}{2}+\frac{5 \theta_{0}^{4}}{2}\right)-D^{2}\left(\frac{2 \theta_{0}^{4}}{3}-2 \theta_{0}^{2}+\frac{5}{3}\right)\right]-\frac{225 \pi^{2}}{256} \frac{1+2 \theta_{0}^{2}}{\theta_{0}\left(\theta_{0}^{2}+1\right)^{3}}-\frac{\theta_{0}}{r_{\Lambda \varepsilon}^{2}\left(\theta_{0}^{2}+1\right)}
$$

In the previous expression the expansion parameters have been rewritten in such a way that $\varepsilon_{\mathrm{E}}=\varepsilon$ and $\varepsilon_{\Lambda}=\varepsilon / r_{\Lambda \varepsilon}$. The cosmological constant changes the angular positions of the images as seen by the observer. The image angular splitting reads

$\vartheta^{+}-\vartheta^{-}=\vartheta_{\mathrm{E}}\left\{\sqrt{\beta^{2}+4}-\frac{15 \pi \beta \varepsilon}{16 \sqrt{\beta^{2}+4}}+\frac{\varepsilon^{2}}{\sqrt{\beta^{2}+4}}\left[16-\frac{225 \pi^{2}\left(\beta^{4}+6 \beta^{2}+6\right)}{256\left(\beta^{2}+4\right)}+28 D \beta^{2}-\frac{8 D^{2}}{3}\left(2+7 \beta^{2}\right)-\frac{2}{r_{\Lambda \varepsilon}^{2}}\right]\right\}$

Deflection angle in gravitational lensing is usually defined in asymptotically flat spacetimes as the angle between the asymptotic tangents to the light ray at the observer and at the source. Despite this definition does not apply to the SdS spacetime, we can identify a sort of contribution of $\Lambda$ to the deflection by comparing the lens equations with and without $\Lambda$. The difference is at order $\varepsilon^{3}$,

$$
\hat{\alpha}_{\Lambda}=-\frac{4 m r_{0} \Lambda}{3 \vartheta}
$$

At a typical angle $\vartheta=\vartheta_{\mathrm{E}}$,

$$
\hat{\alpha}_{\Lambda}\left(\vartheta_{\mathrm{E}}\right)=-\frac{\vartheta_{\mathrm{E}}}{D}\left(\frac{r_{\mathrm{o}}}{r_{\Lambda}}\right)^{2}=-\frac{\vartheta_{\mathrm{E}}}{D} \frac{r_{\mathrm{o}}^{2} \Lambda}{3} .
$$

The expression for the deflection in Eq. (9) differs from the result in [12].

\section{MAGNIFICATION}

The ratio between the angular area of the image in the observer sky and the angular area of the source in absence of lensing gives the (signed) amplification of the image,

$$
\mu=\frac{\sin \vartheta}{\sin B} \frac{d \vartheta}{d B} .
$$

The amplification of the apparent luminosity is then given by correcting by the standard redshift factor. The derivative in 


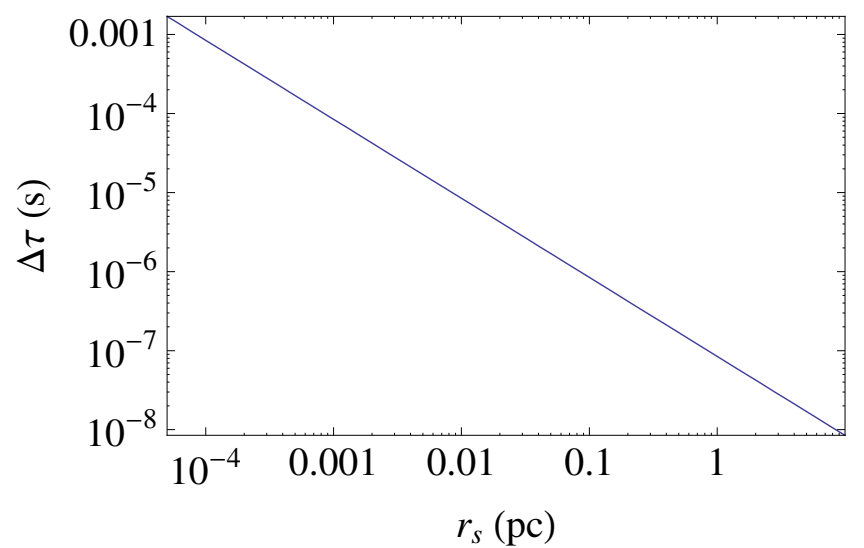

FIG. 1: Time delay (in seconds) due to the cosmological constant between the images of a source behind a Sgr A*-like black hole $\left(m \sim 3.6 \times 10^{6} M_{\odot}, r_{\mathrm{o}} \sim 7.6 \mathrm{Kpc}\right)$ as a function of the source radial distance $r_{\mathrm{s}}$ (in parsecs). The source angular position is fixed at $\beta=1$.
Eq. (10) can be computed through the chain rule by first deriving the coordinate position of the source $\phi_{\mathrm{S}}$ with respect to either $B$ or $\vartheta$ and then combining the results suitably. After multiplying the derivative by the ratio of sines, we introduce the scaled angular variables and rearrange the result as a series in $\varepsilon$,

$$
\mu=\mu_{0}+\mu_{1} \varepsilon+\mu_{2} \varepsilon^{2}+\mathcal{O}\left(\varepsilon^{3}\right) .
$$

The first coefficients of the above expansion series are

$$
\mu_{0}=\frac{\theta_{0}^{4}}{\theta_{0}^{4}-1}
$$

and

$$
\mu_{1}=-\frac{15 \pi \theta_{0}^{3}}{16\left(\theta_{0}^{2}+1\right)^{3}}
$$

The $\Lambda$ correction shows up at the third order,

$\mu_{2}=\frac{8 \theta_{0}^{2}}{\left(1-\theta_{0}^{2}\right)\left(1+\theta_{0}^{2}\right)^{3}}\left\{\theta_{0}^{4}\left(4+2 \theta_{0}^{2}-\frac{675 \pi^{2}}{1024\left(1+\theta_{0}^{2}\right)^{2}}\right)+D \theta_{0}^{2}\left(9-10 \theta_{0}^{2}-5 \theta_{0}^{4}\right)-\frac{D^{2}}{3}\left(1+16 \theta_{0}^{2}-23 \theta_{0}^{4}-12 \theta_{0}^{6}\right)+\frac{\theta_{0}^{2}}{4 r_{\Lambda \varepsilon}^{2}}\right\}$.

Let us consider the microlening case when the two images can not be resolved and the observable is the total magnification $\mu_{\text {tot }}=\left|\mu^{+}\right|+\left|\mu^{-}\right|$. Using the above results, $\mu_{\text {tot }}$ can be written in terms of the unlensed source position as,

$$
\begin{aligned}
\mu_{\mathrm{tot}} & \simeq \frac{\beta^{2}+2}{\beta \sqrt{\beta^{2}+4}}-\frac{15 \pi \varepsilon}{8\left(\beta^{2}+4\right)^{3 / 2}}-\frac{4 \varepsilon^{2}}{\beta\left(\beta^{2}+4\right)^{3 / 2}} \\
& \times\left[\frac{1}{r_{\Lambda \varepsilon}^{2}}+4\left(6+6 \beta^{2}+\beta^{4}\right)-\frac{675 \pi^{2}}{256\left(\beta^{2}+4\right)}\right. \\
& \left.-2 D\left(12+30 \beta^{2}+5 \beta^{4}\right)+\frac{4 D^{2}}{3}\left(18+35 \beta^{2}+6 \beta^{4}\right)\right] .
\end{aligned}
$$

The contribution of $\Lambda$ to the total magnification is negative so that images are slightly de-amplified. The cosmological constant is isotropic and does not perturb the spherical symmetry of the lens. The caustic surface is still a line coincident with the optical axis behind the lens. The tangential critical circle corresponding to the point-like caustics is a perturbed Einstein ring with angular radius

$$
\vartheta_{\mathrm{t}} \simeq \vartheta_{\mathrm{E}}\left\{1+\frac{15 \pi}{32} \varepsilon+\left(4-\frac{4 D^{2}}{3}-\frac{675 \pi^{2}}{2048}-\frac{1}{2 r_{\Lambda \varepsilon}^{2}}\right)\right\} .
$$

Due to $\Lambda$ the area of the Einstein ring slightly decreases.

\section{TIME DELAY}

Light rays corresponding to different images have different travel times. To compute the time delay as measured by an observer we have first to compute the coordinate time $t_{\mathrm{o}}$ when a given ray reaches the observer position and then to translate the difference in coordinate time in proper time. For the SdS metric

$$
t_{\mathrm{o}}= \pm \int f_{\Lambda}(r)^{-1}\left(1-\frac{b^{2}}{r^{2}} f_{\Lambda}(r)\right)^{-1 / 2} d r
$$

where the emission time was consider fixed at $t_{\mathrm{s}}=0$ for all the light rays. Differently from the $r$-motion, the travel time can not be expressed in terms of a new constant of motion $b_{\Lambda}$ that makes the integral in Eq. (12) formally identical to the expression for the Schwarzschild metric. The overall sign in Eq. (12) is adhered to $d r$ to give a positive contribution. As for the geodesic equation, the travel time can be calculated through an expansion in $\epsilon$. We get

$$
\begin{aligned}
t_{\mathrm{o}} & \simeq r_{\mathrm{o}}+r_{\mathrm{s}}+2 m\left(1+\log \frac{4 r_{\mathrm{o}} r_{\mathrm{s}}}{b^{2}}\right)-\frac{b^{2}}{2}\left(\frac{1}{r_{\mathrm{s}}}+\frac{1}{r_{\mathrm{o}}}\right) \\
& +\frac{r_{\mathrm{o}}^{3}+r_{\mathrm{s}}^{3}}{3 r_{\Lambda}^{2}}-\frac{15 m^{2} \pi}{2 b}+\frac{64 m^{3}}{b^{2}}-\frac{b^{4}}{8}\left(\frac{1}{r_{\mathrm{s}}^{3}}+\frac{1}{r_{\mathrm{o}}^{3}}\right) \\
& -4 m^{2}\left(\frac{1}{r_{\mathrm{s}}}+\frac{1}{r_{\mathrm{o}}}\right)+\frac{2 m\left(r_{\mathrm{o}}^{2}+r_{\mathrm{s}}^{2}\right)}{r_{\Lambda}^{2}}+\frac{m^{2}\left(r_{\mathrm{o}}^{3}+r_{\mathrm{s}}^{3}\right)}{2 r_{\Lambda}^{2} b^{2}} .
\end{aligned}
$$

Since an observer measures differences, only terms in the arrival time containing the impact parameter $b$ contribute to the observed time delay, whereas terms depending either only on the radial positions of source and observer or on $m$ and $\Lambda$ do not. Then the term $\sim\left(r_{\mathrm{o}}^{3}+r_{\mathrm{s}}^{3}\right) /\left(3 r_{\Lambda}^{2}\right)$, which is similar to 
a contribution already derived in [5], can not be measured in lensing observations. The measurable time delay is the interval of proper time between the arrivals of the same intrinsic variation in the source luminosity as observed in each of the two images,

$$
\Delta \tau=\sqrt{f_{\Lambda}\left(r_{\mathrm{o}}\right)}\left(t_{\mathrm{o}}^{-}-t_{\mathrm{o}}^{+}\right)
$$

Expanding in $\varepsilon$ and expressing the result in term of the angular source position in absence of the lens, we get

$$
\begin{aligned}
\Delta \tau & =2 m\left\{\delta \tau_{0}+\frac{45 \pi}{8} \varepsilon \sqrt{\beta^{2}+4}+\varepsilon^{2}\left[\frac{1}{2 r_{\Lambda \varepsilon}^{2}}\left(\frac{\left(1+13 D-45 D^{2}+48 D^{3}-16 D^{4}\right) \beta \sqrt{\beta^{2}+4}}{8(1-D)^{3} D}-4 D \delta \tau_{0}\right)\right.\right. \\
& \left.\left.+\frac{4 \beta}{\sqrt{\beta^{2}+4}}\left[8+6 \beta^{2}+\beta^{4}+\frac{1575 \pi^{2}}{1024}\left(3+\beta^{2}\right)+D\left(8-10 \beta^{2}-3 \beta^{4}\right)-\frac{D^{2}}{3}\left(24-14 \beta^{2}-5 \beta^{4}\right)\right]-4 D \delta \tau_{0}\right]\right\}
\end{aligned}
$$

where

$$
\delta \tau_{0}=\beta \sqrt{\beta^{2}+4}+2 \log \frac{\sqrt{\beta^{2}+4}+\beta}{\sqrt{\beta^{2}+4}-\beta} .
$$

Differently from the angular position, the correction term to the time delay due to $\Lambda$ shows factors $D$ and $(1-D)$ at the denominator, so that the effect can be enhanced for sources either very far from $\left(r_{\mathrm{s}} \gg r_{\mathrm{o}}, D \rightarrow 1\right)$ or very near $\left(r_{\mathrm{s}} \ll r_{\mathrm{o}}\right.$, $D \rightarrow 0$ ) to the lens.

\section{NEAR LENSES}

We have seen in the previous sections that the effect of the cosmological constant on lensing observable is really small, being $\sim\left(r_{\mathrm{o}} / r_{\Lambda}\right)^{2}$ times smaller than the main Newtonian term. It can be nevertheless interesting to give some numbers. A classic test of general relativity is measuring the bending of starlight by the Sun. Measurements of the solar deflection using very long baseline interferometry data allowed to put constraints on the deviations from the predictions based on the parametrized post-Newtonian formalism at the level of $\lesssim 0.05 \%[22,23]$. Translating this accuracy into a bound on the cosmological constant, one gets $\Lambda \lesssim 10^{-25} \mathrm{~m}^{-2}$, nearly 17 orders of magnitude worse than the limits obtained from other solar system tests as precession shift and change in mean motion [7].

The supermassive black hole hosted in the radio source Sgr A* in the Galactic center, with a mass of $\sim 3.6 \times 10^{6} M_{\odot}$ and at a distance of $7.6 \mathrm{kpc}$ from the Earth [24], offers another appealing target for testing higher order effects in gravitational lensing with future space- and ground-based experiments $[15,21,25,26]$. For a source $\sim 1 \mathrm{pc}$ behind the black hole, $\Lambda$ induces a variation on the angular position of the images of $\sim 10^{-14}$ arcsec. Accuracies at the level of $\sim 1 \mu \mathrm{arcsec}$, which are within the reach of future missions, are still to low to detect the effect of $\Lambda$. Since multiple images of a single source could be detected behind Sgr A* in the near future, prospects for measurements of time delays can deserve some interest. In Fig. 1 the time delay due to $\Lambda$ for sources behind $\mathrm{Sgr}$ A* is plotted as a function of the source radial distance, with $r_{\mathrm{s}}$ spanning the range from $10 \mathrm{AU}$ to $10 \mathrm{pc}$. For sources very near the black hole, the delay can be as large as $10^{-3} \mathrm{~s}$.

Let us finally consider the impact of the cosmological constant on microlensing analyses. A variation $\delta \vartheta_{\mathrm{E}}$ in the Einstein radius brings a variation of $2 \delta \vartheta_{\mathrm{E}} / \vartheta_{\mathrm{E}}$ in the optical depth. Microlensing events have been observed as far away as in the Andromeda galaxy at $\sim 750 \mathrm{kpc}$ [27]. Due to $\Lambda$, the optical depth would decrease by $\sim 10^{-8}$, which is really negligible.

\section{CONCLUSIONS}

The stagnant theoretical affair between the cosmological constant and the bending of light rays took an hit recently when Rindler and Ishak [12] pointed out how the study of the orbit equation in the coordinate space is not enough to describe the observations of lensing phenomena. This noteworthy criticism has then stimulated some new interest on the subject [13]. In this paper, I have performed an analysis of lensing phenomena in the framework of the SdS metric, which allows a full treatment for systems much smaller than the Hubble radius. I have based my results on a perturbation expansion of the equation of motions, from which I have derived a lens equation accounting for $\Lambda$. The analysis has also showed that the classical argument against $\Lambda$, i.e. that the cosmological constant is dropped out from the exact differential equation for a light path, does not apply to the time delay. It is also to be remarked that the degeneracy between the orbital differential equation in the Schwarzschild metric and that in the SdS spacetime breaks down in presence of a non null angular momentum of the lens.

The argument that $\Lambda$ affects lensing through the metric itself at the observer position is not restricted to the weak deflection limit and applies as well to light rays passing very near to the photon horizon of the black hole. Since SdS null 
geodesics are formally identical to the Schwarzschild case, the calculation of the deflection angle should be performed as usual but the relation between the constant of motion and the observed angle should be revised. However, since the angular separations of the relativistic images are very small with respect to the two primary images, it is customary in the strong deflection limit to neglect higher order corrections.

Even if important on a theoretical point of view, the effect of $\Lambda$ on near lenses, such as the Sun, the supermassive black hole in the Galactic center or compact objects in the halo of near galaxies, is quantitatively very small. Ishak et al. [28] tried to extend the result obtained in the framework of the SdS metric to a cosmological scenario where the distances between lens, source and observer are comparable with the Hubble radius. Some caution should be however used in such an extrapolation. The cosmological lens equation is usually derived combining local results on the light deflection, which are based on an asymptotically flat metric, with considerations on the global geometry of the system and computations of distances, which are on turn based on the global FriedmannLemaitre-Robertson-Walker spacetime in which the system is embedded [29, 30]. As shown in [12] and in the present analysis, both based on the SdS metric, the main contribution of $\Lambda$ to lensing observables comes through the value of the metric at the observer position, which lies in a region of spacetime curved by the cosmological constant. In the classical argument for the cosmological lens equation, local effects are related to a small region in the neighborhood of the lens, and global effects are connected to the large regions of spacetime between source, lens and observer. Then, the contribution of $\Lambda$ as inferred in the SdS framework should be seen as global in the sense that it is connected to the observer radial distance. As far as distances are small with respect the de Sitter horizon, we can safely apply the expressions obtained in the SdS spacetime, but if distances are comparable to the Hubble length then the results should be likely revisited. This will the subject of a future analysis.

A further consideration is that if we are assuming that a constant energy background as the one provided by $\Lambda$ affects lensing, then every other background, such as that provided by dark matter, should have a similar effects. The McVittie metric, which accounts for the presence of a generic cosmological fluid around the central mass and the related expansion of the spacetime, should be used instead of the more specific SdS spacetime and the effect of all the contributions to the cosmological energy budget should be considered even on a small scale [31]. Then, even if $\Lambda$ changes in some ways the expression for the bending angle, the dark matter, whose uniform distribution counteracts the cosmological constant, should nearly compensate in the opposite direction.

The last consideration is that in the case of $\Lambda=0$ the results in this paper updates previous studies for lensing in the Schwarzschild spacetime that were based either on an approximate lens equation, differently from the present analysis which is based on a perturbation analysis of an exact lens equation, or neglected the effect of the metric in the relation between the impact parameter and the observed angle.

\section{Acknowledgments}

M.S. is supported by the Swiss National Science Foundation and by the Tomalla Foundation.
[1] P. J. Peebles and B. Ratra, Reviews of Modern Physics 75, 559 (2003).

[2] P. Jetzer and N. Straumann, Physics Letters B 606, 77 (2005).

[3] J. N. Islam, Physics Letters A 97, 239 (1983).

[4] E. L. Wright, astro-ph/9805292 (1998).

[5] A. W. Kerr, J. C. Hauck, and B. Mashhoon, Classical and Quantum Gravity 20, 2727 (2003).

[6] P. Jetzer and M. Sereno, Phys Rev. D 73, 044015 (2006).

[7] M. Sereno and P. Jetzer, Phys. Rev. D 73, 063004 (2006).

[8] L. Iorio, International Journal of Modern Physics D 15, 473 (2006).

[9] A. Balaguera-Antolínez and M. Nowakowski, Astron. Astrophys. 441, 23 (2005).

[10] A. Balaguera-Antolínez, C. G. Böhmer, and M. Nowakowski, Classical Quantum Gravity 23, 485 (2006).

[11] P. Teerikorpi, A. D. Chernin, and Y. V. Baryshev, Astron. Astrophys. 440, 791 (2005).

[12] W. Rindler and M. Ishak, Phys. Rev. D 76, 043006 (2007).

[13] K. Lake, ArXiv: 0711.0673v1.

[14] P. Bakala, P. Cermak, S. Hledik, Z. Stuchlik, and K. Truparova, ArXiv: (2007), 0709.4274.

[15] C. R. Keeton and A. O. Petters, Phys. Rev. D 72, 104006 (2005).

[16] J. Bodenner and C. M. Will, Am. J. Phys. 71, 770 (2003).

[17] V. Perlick, Phys. Rev. D 69, 064017 (2004).

[18] R. Adler, M. Bazin, and M. Schiffer, Introduction to general relativity (New York: McGraw-Hill, 1965).

[19] W. Rindler, General Relativity (University of Chicago Press, Chicago, 2nd ed., 2006).

[20] G. V. Kraniotis, Classical and Quantum Gravity 22, 4391 (2005).

[21] M. Sereno and F. De Luca, Phys. Rev. D 74, 123009 (2006).

[22] D. E. Lebach, B. E. Corey, I. I. Shapiro, M. I. Ratner, J. C. Webber, A. E. E. Rogers, J. L. Davis, and T. A. Herring, Physical Review Letters 75, 1439 (1995).

[23] S. S. Shapiro, J. L. Davis, D. E. Lebach, and J. S. Gregory, Physical Review Letters 92, 121101 (2004).

[24] F. Eisenhauer, R. Genzel, T. Alexander, R. Abuter, T. Paumard, T. Ott, A. Gilbert, S. Gillessen, M. Horrobin, S. Trippe, et al., Astrophys. J. 628, 246 (2005).

[25] M. Sereno and F. De Luca, ArXiv: 0710.5923.

[26] M. Sereno, Phys. Rev. D 69, 023002 (2004).

[27] S. Calchi Novati, G. Iovane, A. A. Marino, M. Aurière, P. Baillon, A. Bouquet, V. Bozza, M. Capaccioli, S. Capozziello, V. Cardone, et al., Astron. Astroph. 381, 848 (2002).

[28] M. Ishak, W. Rindler, J. Dossett, J. Moldenhauer, and C. Allison, ArXiv: 0710.4726v1.

[29] P. Schneider, J. Ehlers, and E. E. Falco, Gravitational Lenses (Springer-Verlag Berlin Heidelberg New York., 1992).

[30] S. Seitz, P. Schneider, and J. Ehlers, Classical and Quantum Gravity 11, 2345 (1994).

[31] M. Sereno and P. Jetzer, Phys. Rev. D 75, 064031 (2007). 